\title{
Transcutaneous Therapeutic Ultrasound Reduces Infarct Size in a Rabbit Model of Acute Insoluble Ischemic Stroke
}

\author{
Rene Flores ${ }^{1}$, PhD; John D. Lowery ${ }^{2}$, DVM; Robert D. Skinner ${ }^{3}$, PhD; Paula K. Roberson ${ }^{4}$, PhD; Sean D. \\ Woods $^{1}$; William C. Culp ${ }^{1}$, MD
}

Departments of ${ }^{1}$ Radiology, ${ }^{2}$ Laboratory Animal Medicine, ${ }^{3}$ Neurobiology and Developmental Sciences, ${ }^{4}$ Biostatistics, at the University of Arkansas for Medical Sciences, Little Rock, AR 72205

\begin{abstract}
Low-frequency ultrasound (US) enhances muscle and myocardial perfusion without lysis of the arterial obstruction. We evaluated transcutaneous therapeutic ultrasound with tissue plasminogen activator (tPA) for decreases in cerebral infarct volume in rabbits with insoluble arterial occlusions. We hypothesized that US with IPA is effective at reducing infarcts without lysis of the arterial obstruction. New Zealand White rabbits $(n=26 ; 5.2 \pm 0.08$ $\mathrm{kg}$ ) received angiography and three 700 to $900 \mu \mathrm{m}$ embolic spheres were injected into the internal carotid artery occluding its branches. Treatment groups: control $(n=10$; embolized only), tPA alone $(n=7)$, and tPA+US $(n=9)$. Rabbits receiving US received pulsed-wave US (1-MHz, $\left.0.8 \mathrm{~W} / \mathrm{cm}^{2}\right)$ over 1 hour. Rabbits administered tPA received intravenous tPA $(0.9 \mathrm{mg} / \mathrm{kg})$ over 1 hour. Rabbits were sacrificed 24 hours later and infarct volume was determined following staining with triphenyltetrazolium chloride. Immediate collateral flow was determined with digital subtraction angiography. Percent infarct volume was greater $(P=0.035)$ for control $(3.4 \pm 1.0 \%)$ than for tPA+US $(0.9 \pm 1.0 \%)$ rabbits. Rabbits treated with tPA alone $(2.0 \pm 1.1 \%)$ were intermediate and did not differ $(P>0.29)$ from control and tPA+US rabbits. Collateral flow did not $(P=0.93)$ influence infarct volume. Treatment with tPA+US decreases brain infarct volume in rabbits with permanent arterial occlusions, supporting flow studies in muscle or myocardium.
\end{abstract}

Keywords: Collateral flow; ischemic stroke; sonothrombolysis; ultrasound; tPA

\section{Introduction}

Ultrasound (US)-facilitated thrombolysis, or sonothrombolysis, with tissue plasminogen activator (tPA) and 2-MHz transcranial Doppler ultrasound improves arterial recanalization in patients with acute ischemic stroke (Alexandrov et al. 2004). Animal evidence indicates that low-frequency US improves tissue perfusion in the rabbit ischemic limb (40 kHz US) and enhances myocardial perfusion in dogs ( $27 \mathrm{kHz} \mathrm{US})$, even without removing the arterial obstruction (Suchkova et al. 2000; Suchkova et al. 2002; Siegel et al. 2004). As emboli occlude vessels fresh clot forms in stagnant blood upstream and downstream to the point of continued flow. Upstream this is usually to the first major branch that remains open and downstream it is potentially very extensive and continues until collateral flow is encountered. The mechanism of action of improved tissue perfusion due to US administration is not clearly understood but may involve redistribution of collateral flow to the area of ischemia and nitric oxide dependent mechanisms operating at the level of very small vessels (Suchkova et al. 2000; Suchkova et al. 2002). Potential novel therapeutic strategies that improve brain tissue perfusion independent of recanalization would be beneficial in the treatment of acute ischemic stroke, thereby decreasing morbidity associated with stroke due to insoluble occlusions such as embolic atheroma.

Although great emphasis has been given to evaluating the effectiveness of combined TPA and US administration on the lysing of freshly formed clot, understanding the effects of sonothrombolysis with IPA on brain infarct volume following embolization with insoluble emboli is limited (Behrens et al. 2001). Since atheromata, very common embolic agents in ischemic stroke, are insoluble, this has great clinical impact. Perhaps direct effects of ultrasound on collateral vessels with increased dilatation and increased flow into

\section{* Correspondence should be sent to:}

Dr. William C. Culp, Department of Radiology, University of Arkansas for Medical Sciences, 4301 W. Markham St., Slot 556, Little Rock, AR 72205-7199; Email: culpwilliamc@uams.edu; Phone (501) 686-6910; Fax (501) 686-6900 
ischemic areas are responsible here. In order to facilitate selection and management strategies of treatment protocols utilizing tPA and US, a better understanding of sonothrombolysis therapy with tPA with respect to insoluble arterial occlusions is needed. Using a rabbit model of acute insoluble ischemic stroke, the objectives of this study were to (1) investigate the effect of US-facilitated thrombolysis with tPA on infarct volume in rabbits with insoluble arterial occlusions and (2) evaluate the influence of immediate collateral flow on infarct volume using digital subtraction angiography (DSA). The rabbit model of stroke was used because seminal discoveries of the use of tPA which ushered in its use in clinical medicine were made using this model.

\section{Material and Methods}

\subsection{Animals and Surgical Procedures}

All animal procedures were approved by the Institutional Animal Care and Use Committee. New Zealand White rabbits $(n=26$; mean body weight $5.2 \pm 0.08$ $\mathrm{kg}$ ) were used.

The surgical and angiographic procedures were previously described (Culp et al. 2007). Rabbits were sedated with an intramuscular injection of ketamine, $30 \mathrm{mg} / \mathrm{kg}$ (Ketaset; Fort Dodge; Fort Dodge, IA) and xylazine, $3 \mathrm{mg} / \mathrm{kg}$ (AnaSed; Lloyd Laboratories; Shenandoah, IA) and anesthetized with isoflurane (Novaplus; Hospira Inc.; Lake Forrest, IL). A femoral artery was exposed, and utilizing a $3 \mathrm{~F}$ vascular sheath, a modified $65-\mathrm{cm}$ angled-tip 3F catheter (SlipCath; Cook Inc.; Bloomington, IN) was placed into the artery. The 3F catheter was advanced over a 0.025-inch angled-tip guide wire (Glidewire; Terumo Medical Corp.; Elkton, MD) under fluoroscopy to the common carotid artery and subsequently into the internal carotid artery (ICA).

Sub-selective ICA magnification angiography was performed in lateral and frontal projections to document the cerebral vasculature prior to embolization (Fig. 1A). Embolization was accomplished by injection of three 700 to $900 \mu \mathrm{m}$ embolic spheres (Embosphere Microspheres; BioSphere Medical Inc.; Rockland, MA) with 0.7 to $2.0 \mathrm{~mL}$ of saline into the ICA. Flow usually carried the embolic spheres to the middle cerebral artery (MCA; Fig. 2). One minute following embolization, repeat angiography was performed and the location of the arterial occlusion was recorded (Fig. 1B) using a single plane C-arm DSA machine (OEC 9800; GE Healthcare; Salt Lake City, UT). Additional repeat angiography was performed 30 minutes following embolization.

The images obtained from angiography were evaluated for the presence or absence of immediate collateral flow. Collateral flow pathways that were con- sidered were (1) collateral flow through the anterior communicating artery, resulting in retrograde flow in the occluded side toward the affected MCA and (2) posterior to anterior flow through the ipsilateral posterior communicating artery and posterior cerebral artery.

One hour following embolization and angiography, treatment was initiated. An intravenous catheter (Instyle-W; Becton Dickinson; Sandy, UT) placed into an ear vein was used for administration of tPA. Following the procedure the 3F catheter was removed and the incision was sutured. Rabbits were allowed to recover and monitored for adverse effects. Twentyfour hours following embolization, rabbits were sacrificed by an intravenous administration of $1.5-\mathrm{mL}$ of pentobarbital (Euthasol; Virbac Corp.; Fort Worth, $\mathrm{TX})$.

\subsection{Experimental Treatments}

Rabbits were randomly assigned to one of three treatment groups: 1) control $(n=10), 2)$ tPA alone $(n=7)$, or 3$)$ tPA+US ( $n=9)$. Rabbits administered tPA received intravenous tPA (Cathflo Activase; Genentech; South San Francisco, CA) at a dose rate of 0.9 $\mathrm{mg} / \mathrm{kg}$, with $10 \%$ given as a single bolus and the remaining $90 \%$ administered over 60 minutes. Rabbits administered US received transcutaneous pulsedwave (20\% duty cycle) US at $1-\mathrm{MHz}, 0.8 \mathrm{~W} / \mathrm{cm}^{2}$ (Sonicator 716; Mettler Electronics; Anaheim, CA) over 60 minutes. Prior to embolization the right side of the head was clipped of hair and depilatory cream was applied. Following embolization a hand-held $10-\mathrm{cm}^{2}$ therapeutic transducer was placed in front of the ear and behind the eye and was coupled to the skin with US gel. Positioning of the US probe was confirmed fluoroscopically to correspond to the area of the arterial occlusion. Control rabbits were embolized but received no tPA or US.

\subsection{Measurement of Infarct Volume}

Following sacrifice, the brain was harvested and immediately placed in chilled physiological saline for 60 minutes and then sliced coronally at $0.4-\mathrm{cm}$ intervals using a chilled brain mold (RBM-7000C; ASI Instruments Inc.; Warren, MI). Coronal brain sections $(n=8)$ were placed in 1\% 2,3,5-triphenyltetrazolium chloride (Sigma-Aldrich; St. Louis, MO) for 45 minutes at $37^{\circ} \mathrm{C}$ (Bederson et. Al 1986; Brown et al. 2010), fixed in 10\% formalin, and digitally photographed (Fig. 3). Using digital analysis (NIH ImageJ), structures with infarction were identified, measured and recorded. Infarct volume was computed as a percent of the total volume of the brain.

\subsection{Statistical Analysis}

The normal distribution of the variables included in the dataset was tested with the UNIVARIATE proce- 2 - 
dure of SAS (SAS Inst. Inc.; Cary, NC). The ShapiroWilk test for normality showed the infarct volume data not to be normally distributed $(W>0.76, P<0.001)$. Therefore, infarct volume was statistically analyzed with the Kruskal-Wallis test generated with the
NPAR1WAY procedure of SAS. Chi-square analysis, using the FREQ procedure, was used to compare the percentage of rabbits with and without collateral flow among the treatment groups.

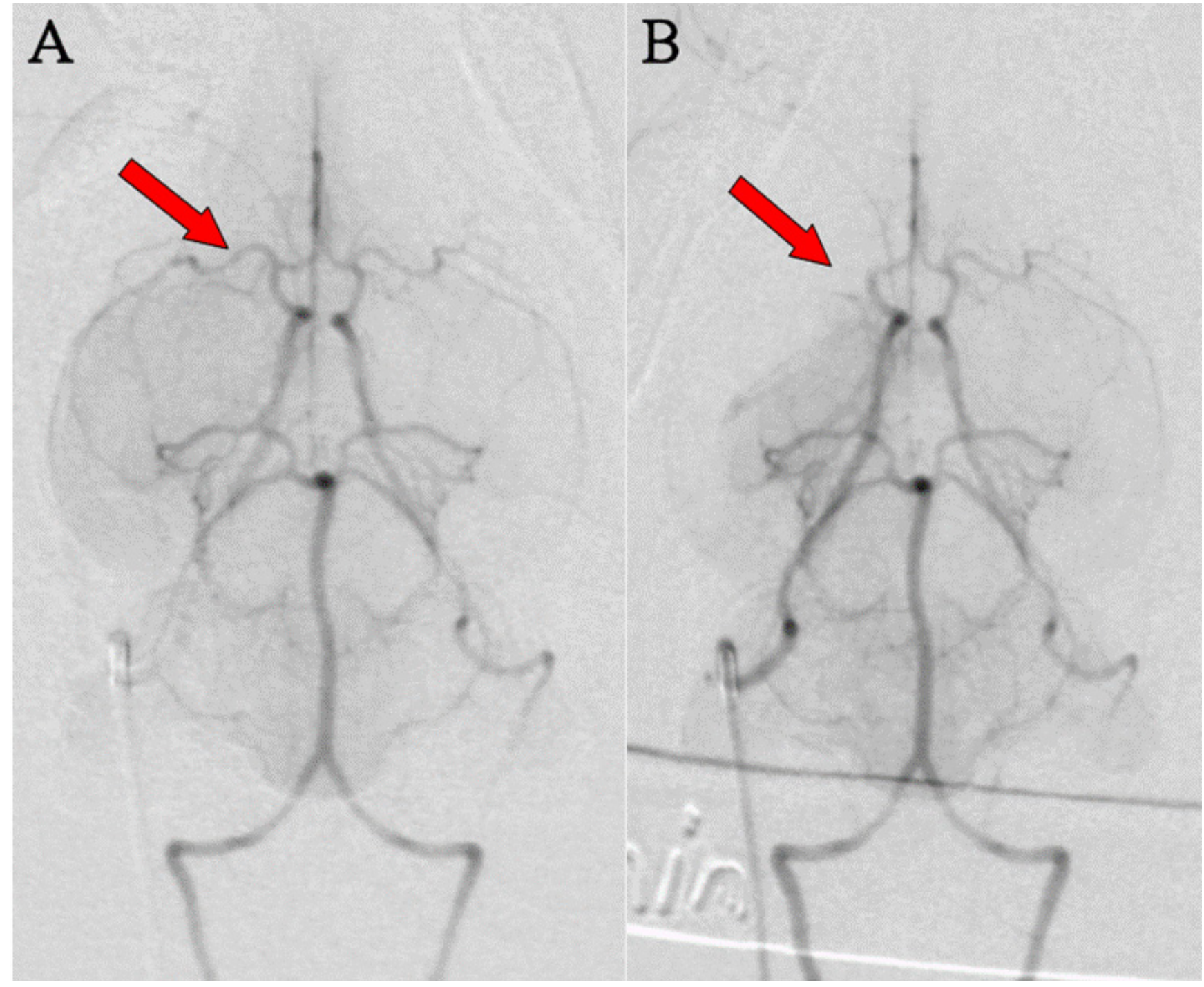

Fig. 1. Selective subtracted cerebral angiogram showing complete filling (arrow) of a middle cerebral artery (MCA) prior to embolization (A). Subtracted angiogram obtained 1 minute following embolization with embolic spheres shows occlusion (arrow) of a MCA (B). 


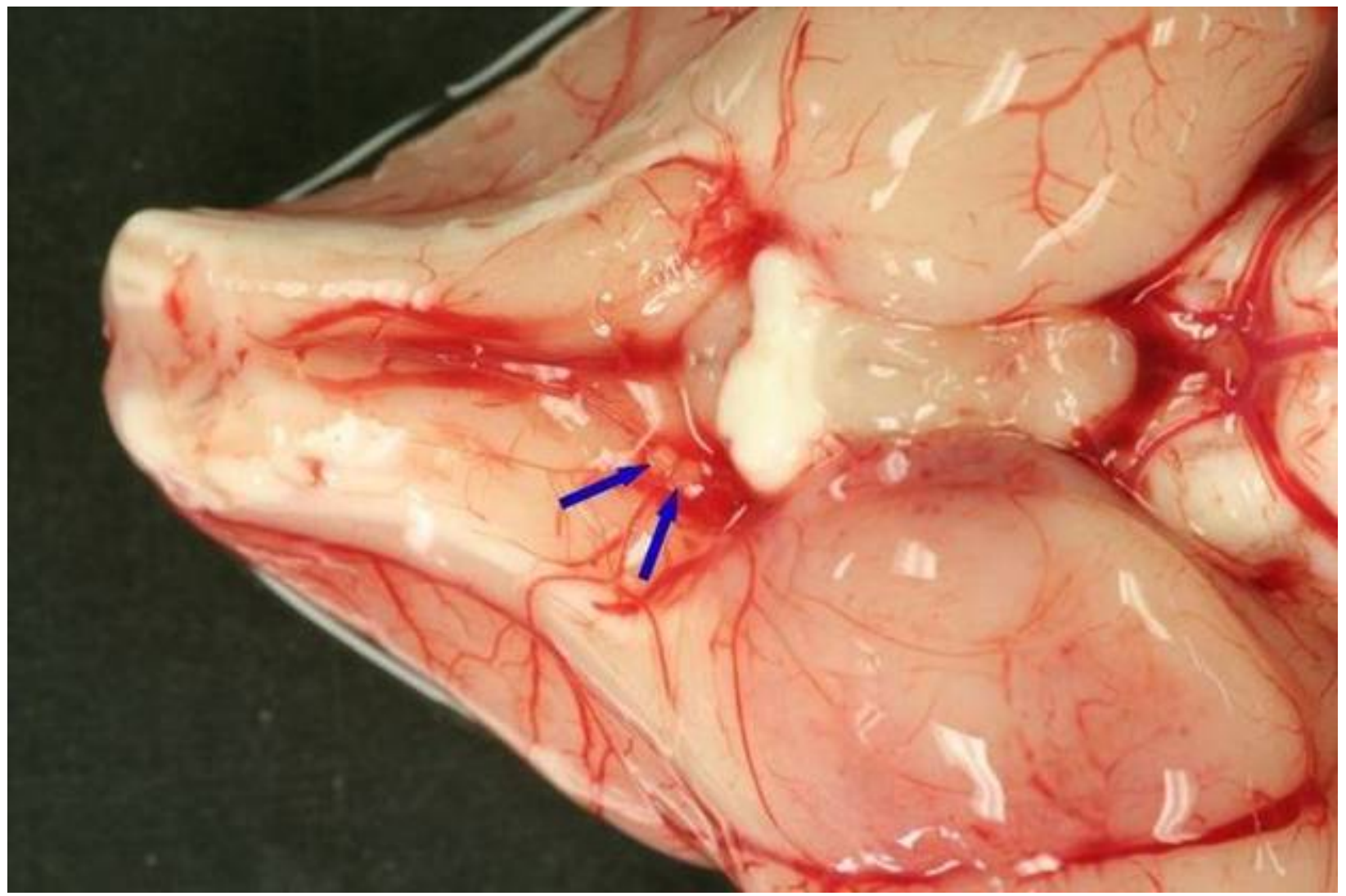

Fig. 2. Ventral view photograph of the brain with embolic spheres visualized (arrows) within the middle cerebral artery (MCA) of rabbits euthanized 24 hours following embolization with spheres. The spheres are localized to the MCA in rabbits undergoing selective embolization by injection of spheres into the internal carotid artery. Anterior is to the left and the brain stem extends to the right of this photograph.

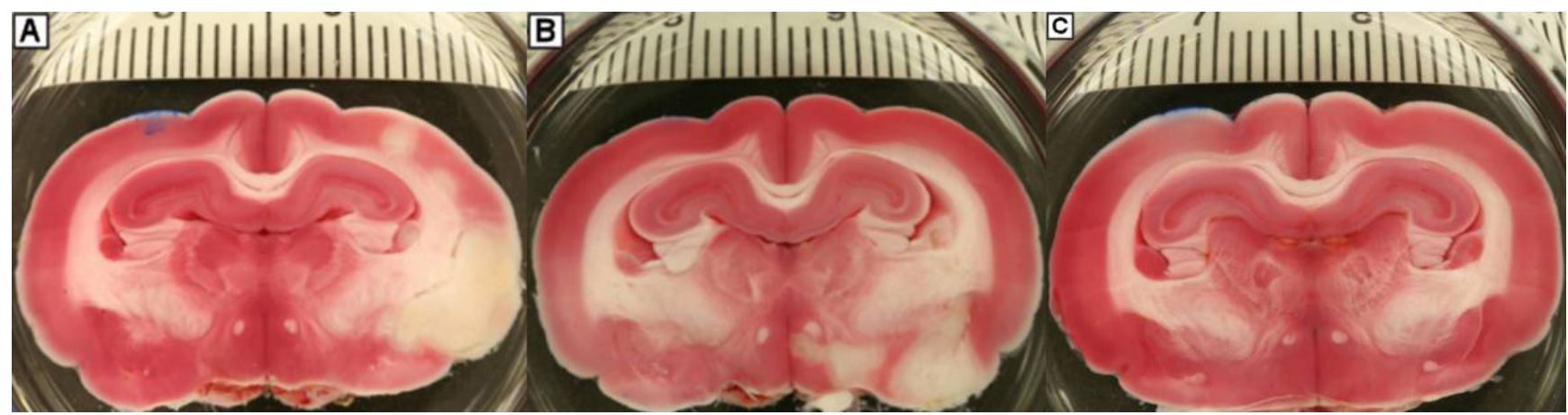

Fig. 3. Representative triphenyltetrazolium chloride-stained brain sections from a control rabbit (A), a rabbit treated with tissue plasminogen activator (tPA) only (B), and a rabbit treated with tPA + ultrasound (C). Control rabbits were embolized but received no tPA or ultrasound. Light colored areas of infarct are readily observed in (A) and (B) while no infarct is observed in (C).

\section{Results}

Percent infarct volume was lower $(P=0.035)$ in rabbits treated with tPA+US $(0.9 \pm 1.0 \%)$ than in control rabbits (3.4 $\pm 1.0 \%$; Fig. 4). Infarct volume was decreased on average but did not differ $(P>0.29)$ be- tween rabbits treated with tPA alone $(2.0 \pm 1.1 \%)$ and control and tPA+US rabbits (Fig. 4). Visible collateral flow did not $(P=0.93)$ influence infarct volume; infarct volume averaged $2.3 \pm 0.8 \%$ and $2.1 \pm 1.1 \%$ for rabbits without and with collateral flow, respectively. Forty -4 - 
percent (4/10), 33\% (3/9), and 14\% (1/7) of control, tPA+US, and tPA alone rabbits, respectively, had evidence of collateral flow on DSA $\left(X^{2}=0.60\right)$ prior to therapy. All of these rabbits were successfully maintained at a normal physiologic state of oxygenation and cardiac function throughout the procedure and treatment.

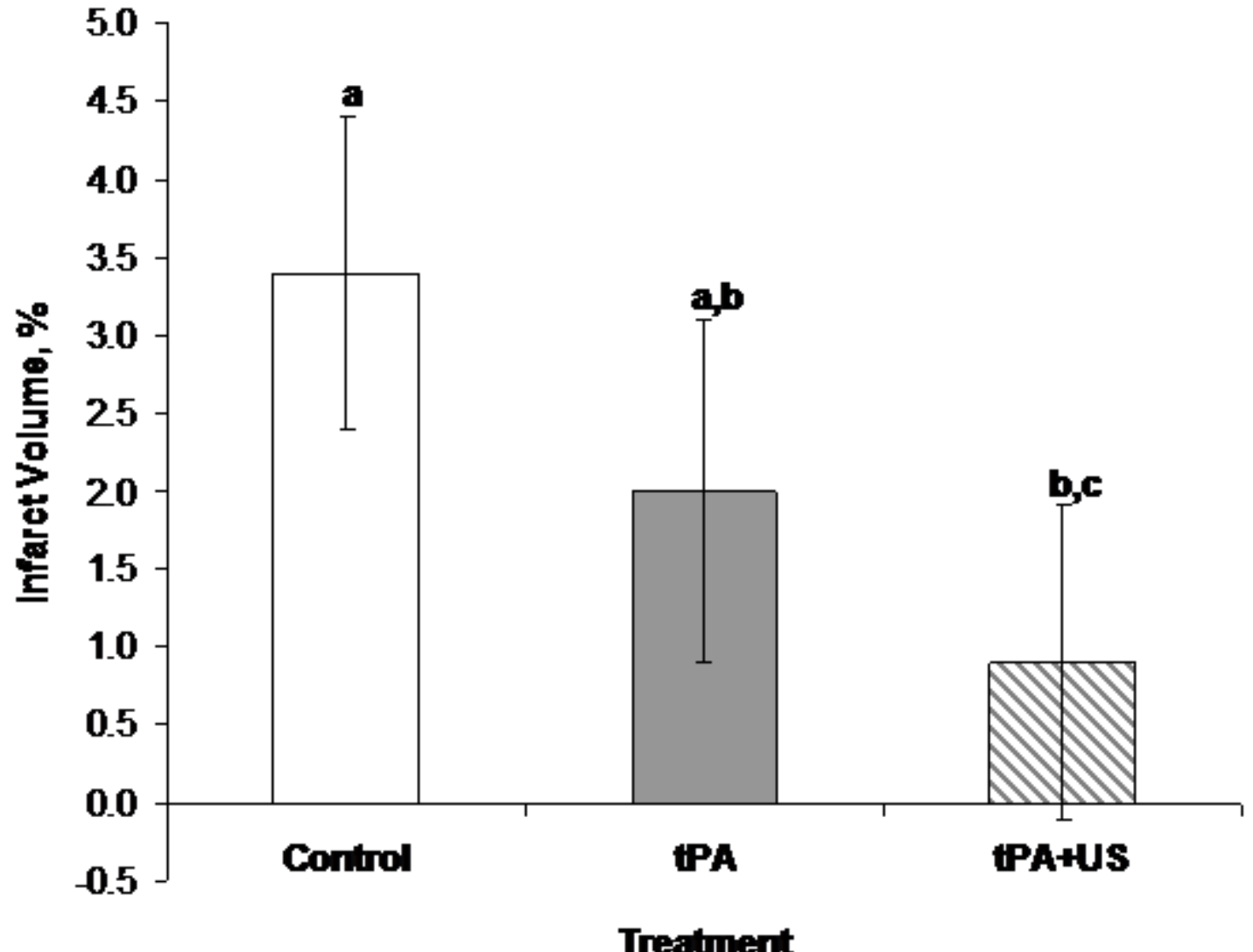

Fig. 4. Percent infarct volume following embolization with embolic spheres and treatment with tissue plasminogen activator (tPA) alone or with tPA + ultrasound (US). Control rabbits were embolized but received no tPA or US. Significantly reduced infarct volumes resulted from tPA+US therapy. ${ }^{a, b, c}$ Means without common superscript letters differ $(P<0.05)$.

\section{Discussion}

Several investigators provided initial studies of USmediated perfusion improvement with permanent occlusions (Suchkova et al. 2000; Suchkova et al. 2002; Siegel et al. 2004). These animal studies demonstrated locally improved transient perfusion values with US application. We use a rabbit model of acute insoluble ischemic stroke and demonstrate that tPA+US decreases infarct volume, an end organ survival change related to perfusion but on a larger scale.

Siegel and colleagues described improved myocardial perfusion in the presence of coronary occlusion using low-frequency $\left(27-\mathrm{kHz} ; 1.4 \mathrm{~W} / \mathrm{cm}^{2}\right)$ pulsed US application for 60 minutes (Siegel et al. 2004). Ultra- sound was directly applied to the exposed heart for 60 minutes after permanent occlusion of the left anterior descending coronary artery. The study measured myocardial perfusion along with myocardial $\mathrm{pH}$ at 30 and 60 minutes after the start of US application. Myocardial perfusion measurements were obtained with a laser Doppler flow meter. The light penetrates the muscle about $1 \mathrm{~mm}$ (Siegel et al. 2004). In the present study, brain infarct volume was determined 24 hours following embolization. This time frame is more reflective of outcome at the whole organ level rather than surface level $\mathrm{pH}$ and tissue perfusion.

Suchkova et al. showed that the application of continuous low-frequency $(40-\mathrm{kHz})$ US to ligated rabbit femoral arteries resulted in improved tissue perfusion -5 - 
and a reversal in acidosis (Suchkova et al. 2002). Enhanced muscle tissue perfusion seems to be mediated through nitric oxide dependent mechanisms. Nitric oxide synthase activity in muscle increased 3.6fold following low-frequency US exposure. Furthermore, pre-treatment with $\mathrm{L}-\mathrm{N} \omega$-nitro-arginine methyl ester, a nitric oxide synthase inhibitor, blocked all US enhanced muscle tissue perfusion. Administration of US produces pressure waves that subsequently impart mechanical energy to tissues, ultimately affecting the endothelial cell mechanoreceptors and resulting in the release of nitric oxide (Suchkova et al. 2002; Siegel et al. 2004, Davies 1995). The study of Suchkova and co-workers utilized $40-\mathrm{kHz}$ at intensities from 0.25 to $0.75 \mathrm{~W} / \mathrm{cm}^{2}$ for 60 minutes (Suchkova et al. 2002). Rabbits in the current study were exposed to pulsed US (1-MHz) at an intensity of $0.8 \mathrm{~W} / \mathrm{cm}^{2}$ for 60 minutes.

The use of $1 \mathrm{MHz}$ US at these parameters is well established in our series of papers (Culp et al. 2002; 2003a; 2003b; 2004, 2007; Brown et al. 2010) and the logic of rabbit dose was based on equaling the US delivered in a successful pig cerebral sonothrombolysis model (Culp et al. 2003a; 2004). We measured the pigs with an intracranial hydrophone and did the same in a rabbit to set the rabbit dose. The distribution of US with this transducer is a simple cylinder with a small central axis peak. It is not focused and even with the rabbit skull intact, the US field completely covers the entire head. While the US beam certainly attenuates with distance and is distorted by the skull, the far side of the brain is well within the nominal effective range of 6 to $8 \mathrm{~cm}$.

Novel treatment strategies that improve brain tissue perfusion independent of recanalization would be beneficial in the treatment of acute ischemic stroke, a disease without reliable high yield treatments at this time. Using embolic spheres to occlude the MCA of rabbits provides an approach to enhance our understanding of the physiologic effects of sonothrombolysis with tPA without reestablishment of large vessel flow. Although we did not measure nitric oxide synthase activity, it may be hypothesized that rabbits receiving US in the present study may have had increased synthase activity in brain tissue or vascular elements exposed to US waves. This might explain differences observed between control rabbits and rabbits treated with tPA+US with respect to infarct volume. Other possible mechanisms such as heating and physical oscillations of the tissue must also be considered, but these factors were not measured in this study. However, Hardig et al. (2003) noted that US alone did not worsen ischemic damage in the rat brain embolized in the MCA. The role of TPA is also of importance. Apparently, secondary clots upstream and downstream of insoluble emboli are lysed by
tPA+US favoring improved primary or collateral flow and ultimate patency. Results herein provide evidence that IPA when administered in combination with US decreases infarct volume, supporting the transient improvements observed following US application in muscle (Suchkova et al. 2000) and myocardium (Siegel et al. 2004), and achieving the first objective of this study.

As a secondary objective of this study, we assessed the influence of immediate collateral flow seen on DSA on infarct volume as a means to better understand physiological mechanisms that may influence infarct volume in embolized rabbits. Redistribution of collateral flow in the area of ischemia has been proposed as a possible mechanism of improved tissue perfusion (Suchkova et al. 2000). Infarct volume did not differ between rabbits having visible collateral flow on DSA and rabbits without collateral flow. However, it should be emphasized that visible collateral flow only reflects immediate collaterals seen in the first 30 minutes and not those acquired over longer times due to therapy or physiological processes. Angiography also will not define very small vessels which may be critical to collateral development. Repeat sub-selective angiography following therapy in similar animals was found to trigger arterial spasm in those ischemic brains; this angiography failed to show collaterals well and worsened outcomes. This technique was not included in this study. Nevertheless, the animal stroke model used in the current study provides initial evidence to suggest that mechanisms other than simple collateral flow are involved through which US in combination with tPA decreases infarct volume. Thrombolysis due to tPA and US, and vasodilatation perhaps due to nitric oxide mechanisms, both seem likely to contribute to opening arterioles, capillaries, and very small veins here on the microscopic scale, a scale not visible on angiography.

While improved arterial flow is taken as an excellent indicator of expected clinical improvement in human studies (Alexandrov et al. 2004) the effects shown in our study without large artery clearing may indicate other beneficial actions from the combination of tPA and US, possibly on a much smaller scale. It may be important to open smaller vessels as well as the larger ones to prevent repeat occlusion in ischemic brain. Repeat occlusion is a common occurrence following therapy that shows initial success with large artery thrombus. If this technique maintains flow and prevents additional clot formation for a short time after the start of ischemia, the nitric oxide mechanism and other vasodilatation may be enough to maintain flow after US application and lead to less thrombosis and infarction.

-6 -

J Exp Stroke Transl Med (2011) 4(1): 1-7

Society for Experimental Stroke (www.s4es.org) 
Limitations include the lack of angiography to demonstrate late collateral development following therapy, as well as, the need for better understanding of the mechanisms involved. Future studies will investigate the role of nitric oxide in protecting brain tissue in this model. These results are based on the direct measurement of stroke volumes, a 24-hour TTC tissue stain reflective of basic brain infarction (Bederson et al. 1986). Although this method has been used extensively for identification and size determination of cerebral infarcts in various animal models, it is not without limitations. The possibility that TTC will stain tissue that has been subject to ischemia, and thus fail to distinguish infarcted brain, must be considered. In this study, brain sections were also analyzed with a standard hematoxylin and eosin stain and the infarct areas were correlated (data not shown).

In conclusion, results from the current study with insoluble emboli indicate decreases in infarct volume following treatment with tPA+US. Simple tPA treatment failed to provide significant improvement. The mechanism of action whereby treatment decreases infarct volume in the current animal model appears to not involve immediate large vessel collateral flow.

\section{Acknowledgement}

This work was supported by NIH grant R01 HL092481

\section{Conflict of interest}

None

\section{References}

Alexandrov AV, Molina CA, Grotta JC, Garami Z, Ford SR, Alvarez-Sabin J, Montaner J, Saqqur M, Demchuk AM, Moye LA, Hill MD, Wojner AW, CLOTBUST Investigators. Ultrasound-enhanced systemic thrombolysis for acute ischemic stroke. N Engl J Med 2004;351:21702178.

Bederson JB, Pitts LH, Germano SM, Nishimura MC, Davis RL, Bartkowski HM. Evaluation of 2,3,5triphenyltetrazolium chloride as a stain for detection and quantification of experimental cerebral infarction in rats. Stroke 1986;17:1304-1308.

Behrens S, Spengos K, Daffertshofer M, Wirth S, Hennerici M. Potential use of therapeutic ultrasound in ischemic stroke treatment. Echocardiography 2001;18:259-263.

Brown AT, Skinner RD, Flores R, Hennings L, Borrelli MJ, Lowery J, Culp W. Stroke location and brain function in an embolic rabbit stroke model, J Vasc Interv Radiol. 2010;21:903-909.

Culp BC, Brown AT, Erdem E, Lowery J, Culp WC. Selective intracranial magnification angiography of the rabbit: basic techniques and anatomy. J Vasc Interv Radiol 2007;18:187-192.

Culp, WC, Erdem, E, Roberson, PK, Husain, MM. Microbubble Potentiated Ultrasound as a Method of Stroke Therapy in a Pig Model: Preliminary Findings. J Vasc Interv Radiol 2003a;14:1433-1437.

Culp, WC, Porter, TR, Lowery, J, Xie, F, Roberson, PK, Marky L. Intracranial Clot Lysis with Intravenous Microbubbles and Transcranial Ultrasound in Swine. Stroke 2004;35:2407-2411.

Culp, WC, Porter, TR, McCowan, TC, Roberson, PK, James, CA, Matchett, WJ, Moursi, M. Microbubble Augmented Ultrasound Declotting of Thrombosed Dialysis Grafts in Dogs. J Vasc Interv Radiol 2003b;14:343-347.

Culp, WC, Porter, TR, Xie, F, Goertzen, TC, McCowan, TC, Vonk, BN, Baxter, BT. Microbubble Potentiated Ultrasound as a Method of Declotting Thrombosed Dialysis Grafts. Cardio Vasc Interv Radiol 2002;24:407-412.

Davies PF. Flow-mediated endothelial mechanotransduction. Physiol Rev 1995;75:519-560.

Hardig BM, Persson HW, Gido G, Olsson SB. Does lowenergy ultrasound, known to enhance thrombolysis, affect the size of ischemic brain damage? J Ultrasound Med. 2003;22:1301-1308.

Meairs S, Culp WC. Microbubbles for Thrombolysis of Acute Stroke. Cerebrovasc Dis 2009 27S:55-65

Siegel RJ, Suchkova VN, Miyamoto T, Luo H, Baggs RB, Neuman Y, Horzewski M, Suorsa V, Kobal S, Thompson $\mathrm{T}$, Echt $\mathrm{D}$, Francis CW. Ultrasound energy improves myocardial perfusion in the presence of coronary occlusion. J Am Coll Cardiol 2004;44:1454-1458.

Suchkova VN, Baggs RB, Francis CW. Effect of $40-\mathrm{kHz}$ ultrasound on acute thrombotic ischemia in a rabbit femoral artery thrombosis model: enhancement of thrombolysis and improvement in capillary muscle perfusion. Circulation 2000;101:2296-2301.

Suchkova VN, Baggs RB, Sahni SK, Francis CW. (2002) Ultrasound improves tissue perfusion in ischemic tissue through a nitric oxide dependent mechanism. Thromb Haemost 2002;88:865-870.

Tsivgoulis, G, Alexandrov, A, Culp, WC. Ultrasound Enhanced Thrombolysis in Acute Arterial Ischemia. Ultrasonics 2008;48:303-311. 